\title{
Wild and native plants and mushrooms sold in the open-air markets of south- eastern Poland
}

\author{
Renata Kasper-Pakosz ${ }^{1}$, Marcin Pietras ${ }^{2,3}$ and $Ł u k a s z ~ Ł u c z a j j^{4^{*}}$
}

\begin{abstract}
Background: The study of plants and fungi sold in open-air markets is an important part of ethnobotanical enquiry. Only few such studies were carried out in Europe.

Methods: Four of the largest open-air markets of south-eastern Poland were visited regularly, and the plants sold in them were recorded between 2013 and 2015. The aim of the study was to record native and/or wild species sold in the markets. All the plants sold in the markets were photographed regularly. In each market, 25 sellers were interviewed. Voucher specimens were collected and fungi were identified using DNA barcoding.

Results: Altogether, 468 species of plants were recorded, 117 of them native to south-eastern Poland - 19 only collected from the wild and 11 both wild and cultivated. Seventeen of the species are under legal protection. Most protected plants were sold from cultivation, although proper authorization procedures had not been performed. Thirty-two species of fungi were sold (including two cultivated species), all of them for culinary purposes. Two species (Lactarius quieticolor, Leccinum schistophilum) are new to the mycobiota of Poland.

Ornamental plants constituted a large section of the market, and they dominated the group of native species. Food plants dominated among wild-collected plants and were sold mainly as fruits for jams, juices and alcoholic drinks, or as culinary herbs. Very few medicinal or green vegetable plants were sold. An interesting feature of the markets was the sale of Ledum palustre as an insect repellent.

Conclusions: Finding two species of fungi which are new to Poland highlights the importance of DNA barcoding in ethnomycological studies. Most items in the markets are ornamental plants, or edible fruits and mushrooms. Very few medicinal plants and green vegetables are sold, which differentiates the markets from southern European ones. Such a pattern is probably the model for most central European markets.
\end{abstract}

Keywords: Ethnobotany, Ethnomycology, DNA bar-coding, Protected plants, Medicinal plants, Conservation, Fungi

\section{Background}

The study of plants sold in open-air markets is an important part of ethnobotanical enquiry [1, 2]. Plants which are sold in such places are usually those which are culturally the most salient. In traditional agricultural societies the market is often the main source of goods sold and bought by villagers. Even with the advent of regular shops and supermarkets in more modernized societies, open-air markets remain an important centre of

\footnotetext{
* Correspondence: lukasz.luczaj@interia.pl

${ }^{4}$ Department of Botany, Institute of Applied Biotechnology and Basic

Sciences, University of Rzeszów, Werynia 502, 36-100 Kolbuszowa, Poland

Full list of author information is available at the end of the article
}

plant commerce for both urban and rural dwellers. Many of the plants sold in the markets come from the wild, thus these places are inherently connected with the issue of sustainable collection of plant material from wild growing populations.

Rich traditions of the sale of plants in markets still persist in Europe, in spite of its high level of modernization. Probably the first regular studies of the ethnobotany of markets in the world, or at least in Europe, were performed by Polish scholars. In 1927 Muszyński [3] made a list of medicinal plants sold in the market of Vilnius (then Poland, nowadays Lithuania). Very soon after, in 1933, Jerzy Wojciech Szulczewski, a 
local biologist and ethnographer, issued a paper containing a detailed list of medicinal plants, and another about edible and medicinal fungi $[4,5]$ (later reprinted in [6]) sold in the markets of Poznań, the largest city of western Poland. Just a few years later Szulczewski recorded 56 species of edible and medicinal fungi and 79 species of medicinal plants sold in these markets. His study was revisited in 2013 and a dramatic reduction in the sales of medicinal plants was observed [7].

Karousou et al. [8] studied medicinal herbs sold on 15 stalls scattered through markets in the three largest cities in Cyprus. A total of 57 taxa were recorded, of which 32 were cultivated and 14 wild. Similarly, Hanlidou et al. [9] studied medicinal plants in Thessaloniki, Greece. The majority (131) of the 172 recorded taxa were of local origin.

Łuczaj et al. [10] studied wild edible greens sold at 11 town markets in Dalmatia (on the southern coast of Croatia). According to the authors, the use of wild green vegetables (leaves, buds, stems) is very widespread in the Mediterranean. In total, 37 species were recorded.

Probably the longest list of plants sold in local markets was recorded by Ertug [11], in the Bodrum area of Turkey, who recorded 390 species on sale. Most of the recorded plants were wild edible plants, although plants used for fodder, medicine or crafts were also noted. Another study which recorded wild food plants in Turkey was carried out by Dogan et al. [12] who surveyed 18 markets in Izmir and found that 46 species of wild edible plants were sold. Nedelcheva and Dogan studied open-air markets on both sides of the Bulgarian-Turkish border. They found that predominantly medicinal plants are sold in Bulgarian markets, whereas in Turkish markets there are many more wild vegetables sold $[13,14]$.

A number of scholars have researched plants sold in Asian markets. For instance Pemberton et al. [15] surveyed the three largest markets of wild edible and medicinal plants in Seoul, South Korea. Xu et al. [16] and X [17] looked at plants sold in Xishuangbanna, in the tropical part of Yunnan, China. Shirai \& Rambo [18] presented the results of research on wild species sold on the main town square in Khon Kaen, in north - eastern Thailand. The diversity of wild species sold there is high; much higher in the rainy season than in the dry season. They found 60 wild species, of which 54 were plants, and 6 mushrooms. Konsam et al. [19] found a large diversity of wild vegetables sold in the markets of Manipur, India. The ethnobotany of open-air markets was also studied in Pakistan, Iraq, Iran and Kyrgyzstan [20-23].

One of the earliest ethnobotanical works on markets is by Bye [1] who recorded medicinal plants sold in three cities in northern Mexico. Several other authors researched the ethnobotany of markets in South and Central America [24]. For example De Albuquerque et al. [25] compared lists of plants sold in the North-East of Brazil in the city of Recife, the capital of Pernambuco state. Between 1995 and 2002 the number of plants sold increased from 58 to 136 species. Many of the plants are used for medicine, but they often have magical or hygienic connotations. A similar dominance of medicinal plants is found in the markets of Bolivia [26].

African markets are also dominated by medicinal plants (see e.g. [27-30]). Ouarghidi et al. [31] recorded medicinal plants sold in the three markets of Marrakech. They found that many of the species were falsifications and actually, false cheaper species are sold under different names. The fact that many important medicinal roots are not readily available in the markets of Marrakech suggests that these wild species may be in danger of extinction, and the scarcity and high demand for some species has led to their replacement by other taxa. The article lists species that are sold as replacements or forgeries. Similar results were obtained by Kool et al. [32] who found several rare and endangered species in the markets of southern Morocco.

In practically all of the above-mentioned studies from around the world the number of wild species sold is higher than the number of cultivated plants. Some authors raise the question to what extent such commerce endangers wild populations [33]. This is, for example, the case in Morocco concerning medicinal plants. The trade of ornamental plants, for example orchids, may also endanger local populations [34].

Another issue is the sale of mushrooms in open-air markets. It is widespread in many countries of the globe and often regulated [35-37], but the taxa which are sold are often not properly documented, due to the lack of voucher specimens. Recent advances in DNA barcoding techniques for fungi enable a more accurate identification of the species $[38,39]$.

As previously mentioned, the issue of plant commodification is also connected with conservation status plants are often protected because they are attractive, useful and prone to extinction. Although the first plant protected by law in Poland was the yew-tree (in 1420 by king Władysław Jagiełło - [40]), nowadays a large proportion of protected plants are those which have attractive flowers which make them prone to being picked or dug out for private gardens or for sale [41], for example Galanthus nivalis, Leucojum vernum, Orchidaceae, Lilium martagon etc. Such flowers formed the core of protected plants in the first post-World War II law on plant protection in Poland [42]. In Poland the overharvesting of wild medicinal plants used to be a problem, and rare medicinal plants constituted another large sector of protected plants. They were usually partially protected so that the authorities could license their gathering. Some of the species were not very rare at all 
(e.g. Asarum europaeum, Viburnum opulus, Frangula alnus) and their protection was cancelled in the most recent plant protection legislation [41], as the gathering of medicinal plants is much less widespread now. However, much earlier, at the end of the 19th century, many populations of highland alpine plants in the Tatras were decimated by pickers supplying medicinal "roots" to herbalists [43].

The aim of this study was to record wild plants and fungi sold in the markets of south-eastern Poland. Our hypotheses were:

1. The main wild products sold in the markets are ornamentals, wild fruits and edible fungi. Wild vegetables are not sold in the markets. This is the pattern of consumption of wild foods in Poland, and we expected it to be reflected by the choice of plants in the markets.

2. Some protected and rare wild plants are sold.

3. Few medicinal plants are sold due to the general decrease in gathering activities.

\section{Methods}

The research was performed following the code of ethics of the American Anthropological Association [44] and the International Society of Ethnobiology Code of Ethics [45]. Oral prior informed consent was acquired.

Four open-air markets from southeastern Poland were selected for the study (Fig. 1). This included probably the largest market in this part of Poland, in Rzeszów (190,000 inhabitants), the capital of Podkarpacie region. The other three markets were located in three (out of 21) county towns in the region - Jarosław (ca. 39,000 inhabitants), Leżajsk $(14,000)$ and Przemyśl $(63,000)$.
Open-air markets are located in most towns in Poland. In the past (e.g. before World War II) they were placed in a central market square (a typical feature of Polish medieval towns), but nowadays they were gradually relocated to less central locations. Once the centers of all commerce in towns, including selling animals, now they are a mixture of stalls with cheap clothes, tools, agricultural products, plants and mushrooms.

Data were collected in two ways. The first approach consisted of regular observations of markets. The markets of Rzeszów, Jarosław and Leżajsk (Fig. 1) were visited every week on the days when most people come to buy products ( $\mathrm{J}$ and $\mathrm{L}$ on Tuesday and $\mathrm{R}$ on Saturday) in the periods of September-October 2013, and from the end of February to the end of October 2014 and 2015. In 2015, the markets were visited only once every two weeks. Additionally the market in Przemyśl was visited seven times in 2015, from April to October, at monthly intervals. Altogether 13,488 photos were taken. Photographic documentation enabled the quick recording of plants used in public spaces and detailed identification of most taxa at least to genus level [46].

Photographs were taken on every visit in order to capture the diversity of cultivated and wild plants sold in the markets (Figs. 2, 3, 4, 5, 6, 7, 8 and 9). Lists of species sold were then made for each visit. Voucher specimens were collected if possible. Unfortunately, usually only parts of plants were available as vouchers and sometimes sellers refused to donate even parts of the plants they sold. The second part of the study consisted of interviews with the plant sellers. Altogether, 100 interviews were conducted ( 25 in each of the four markets). The interviews were carried out in the markets with the owners of plant stalls. The mean age of sellers was 55

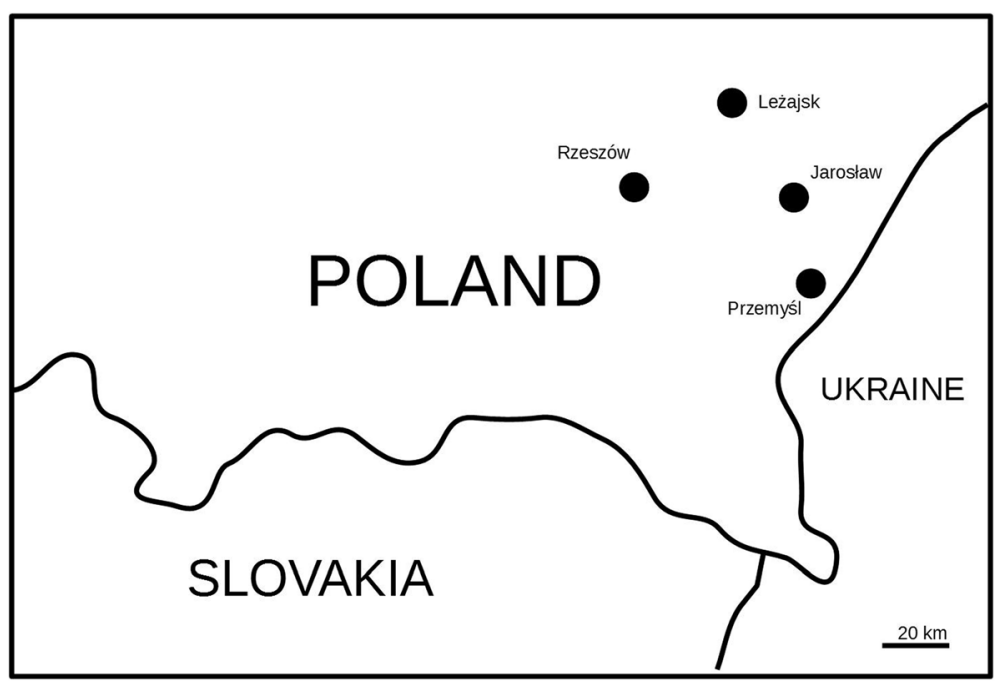

Fig. 1 Studied markets 


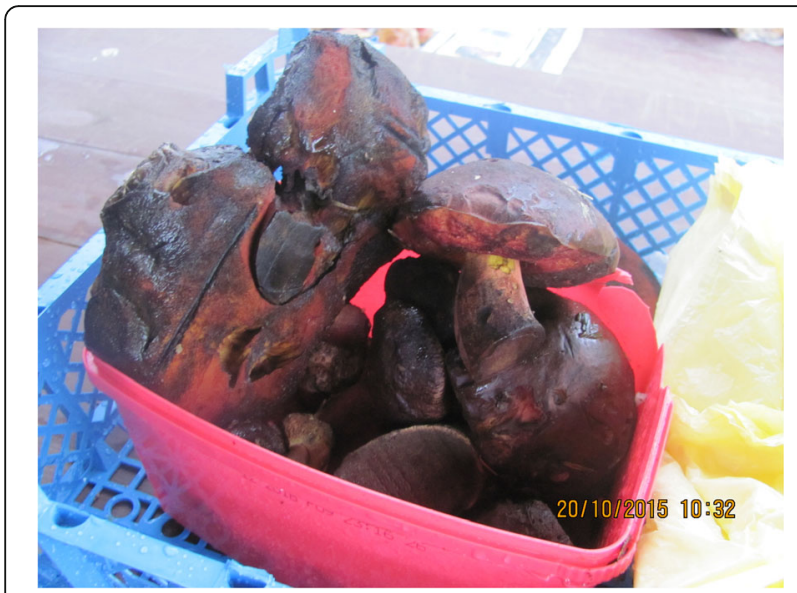

Fig. 2 Boletus luridiformis is widely sold in SE Poland, though its sale is not permitted in open-air market places (photo from Przemyśl)

(median 58.5). The oldest seller was 83, the youngest 22 . There were 62 women and 38 men in the interviewed group. Around half of the sellers were farmers, the rest - a variety of professionals, often retired. Profit and hobby were equally frequently cited motivations for selling plants in the market. The sellers had been selling plants for an average of 19 years (maximum selling time 50 years). As many as $62 \%$ of sellers came every day, and the remainder 3-4 times a week.

The origins of the species (cultivation versus wild) were established based on interviews and the containers in which the species were sold. For example, species sold in small pots without any weeds and with wellestablished roots and one shoot were treated as cultivated, whereas species sold in plastic bags, dug out, with traces of natural vegetation (e.g. woodland mosses, other woodland or semi-natural grassland plants), were suspected to be collected from the wild (although they were sometimes dug out from gardens but then there were no

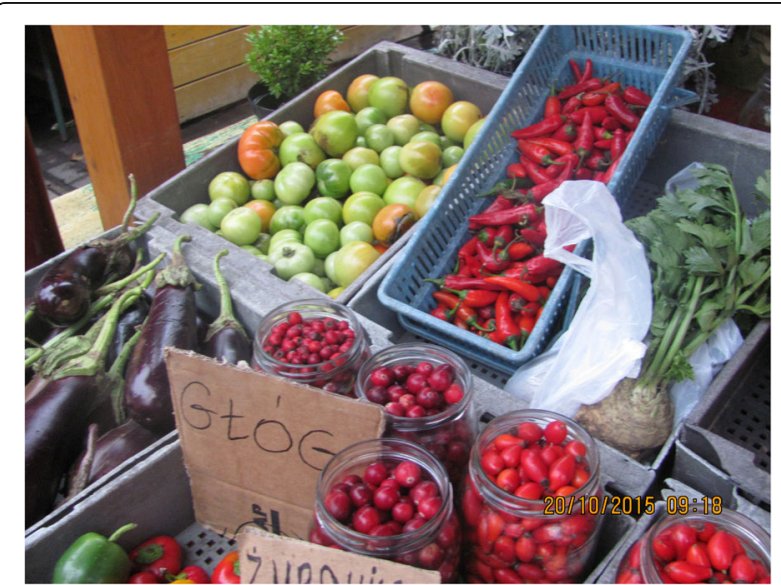

Fig. 3 Rosa, Crataegus and Oxycoccus fruits on sale in Jarosław

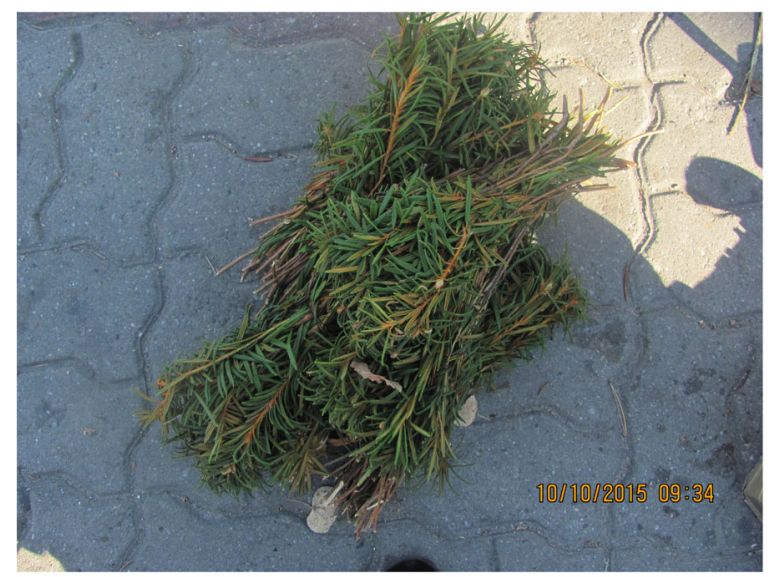

Fig. 4 Ledum palustre is commonly sold as insect repellent in Rzeszów, in spite of being protected by law

traces of forest vegetation). In most cases the answers of the respondents were treated as trustworthy, with the exception of protected or believed-to-be-protected plants - sometimes sellers hesitated or gave very unclear answers when asked if the plant was dug out from the wild. In the case of strange behaviour from informants, we treated the plant as originating from the wild.

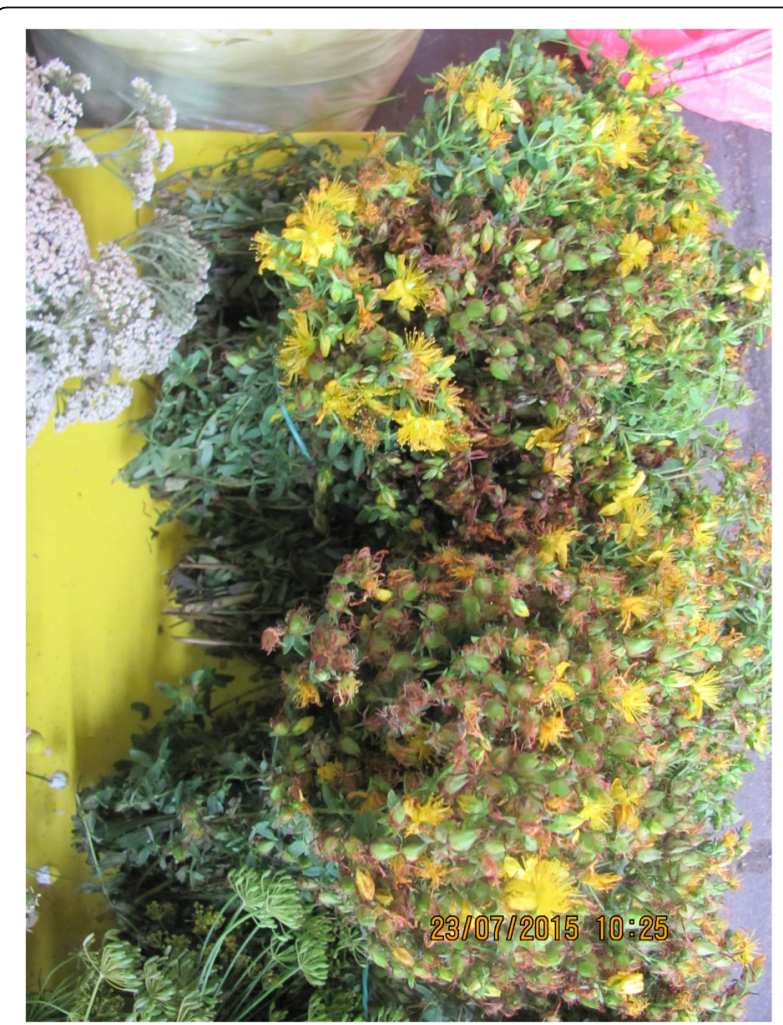

Fig. 5 Hypericum perforatum is one of the very few purely medicinal plants sold in open-air markets in Poland 


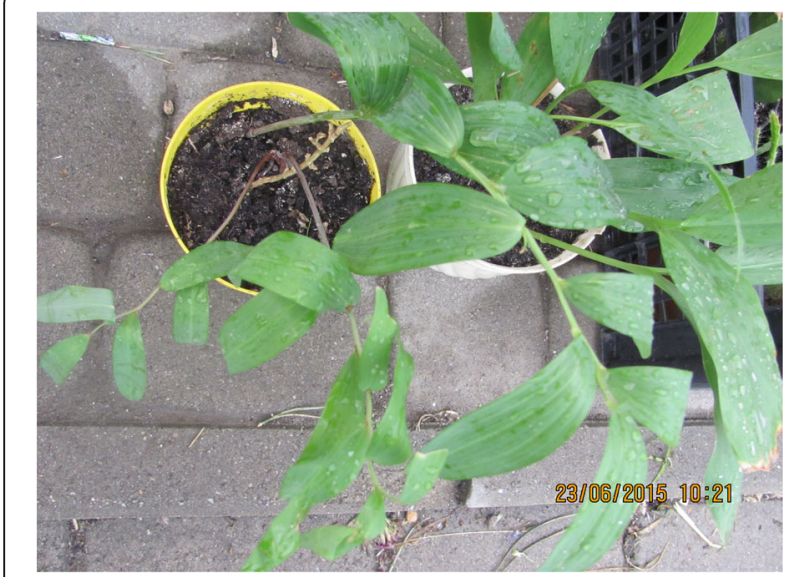

Fig. 6 Wild-origin Polygonatum multiflorum sold as garden ornamental in Przemyśl

Voucher specimens of plants and fungi were deposited in the herbarium of the University of Warsaw (WA). Plants were identified using the standard identification key concerning local floras, and their names follow the Plant List [47]. The status of the plants in the region (native versus non-native established species) was checked with the atlas of the distribution of vascular plants in Poland [48] and other publications on the local flora. Fungi names follow the Index Fungorum [49].

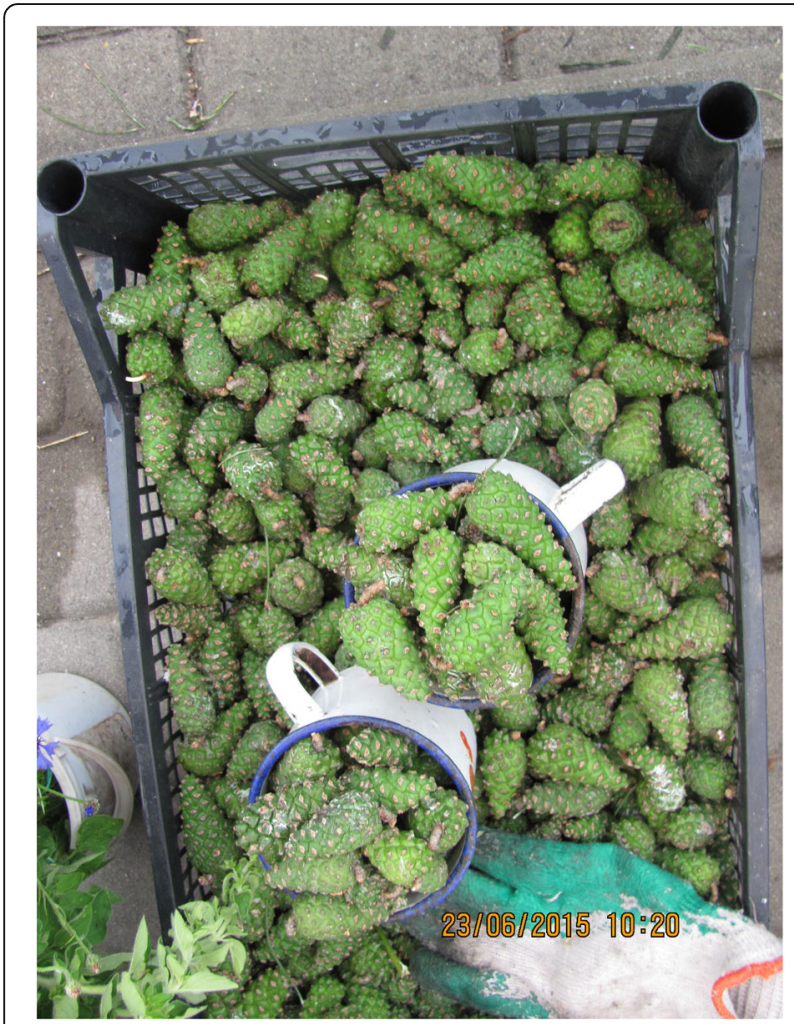

Fig. 7 Young pine cones sold for cough syrup in Przemyśl

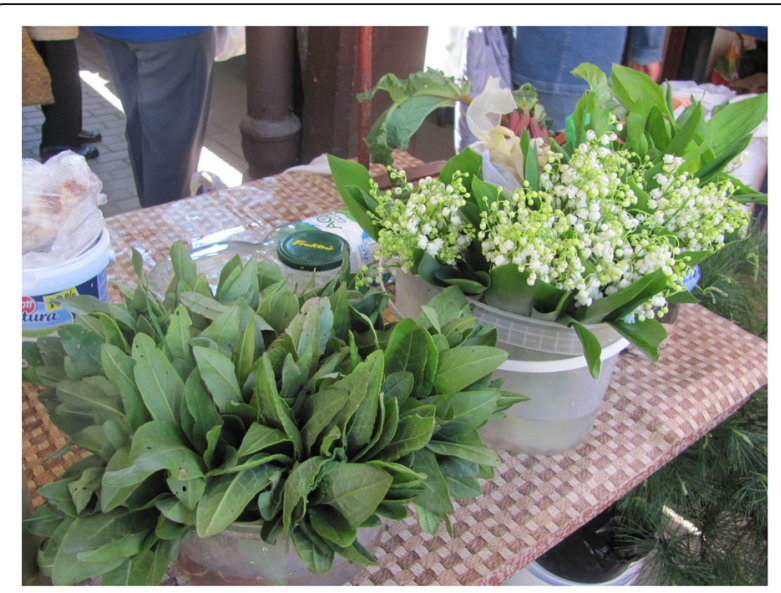

Fig. 8 Convallaria majalis bouquets and Rumex acetosa bunches on sale in Rzeszów

Most fungi specimens were successfully identified using the DNA barcoding technique [50, 51]. Fungal DNA was extracted from a small part of each fruiting body using a Plant and Fungi DNA Purification Kit (Eurx), following standard protocol. The PCR cocktail was composed of $4 \mathrm{ml}$ DNA extract, $0.5 \mathrm{ml}$ each of the primers (ITS5 and ITS4 in $10 \mathrm{nmol}$ concentration) and $5 \mathrm{ml}$ Type-it Microsatellite PCR Kit (Qiagen). PCR was

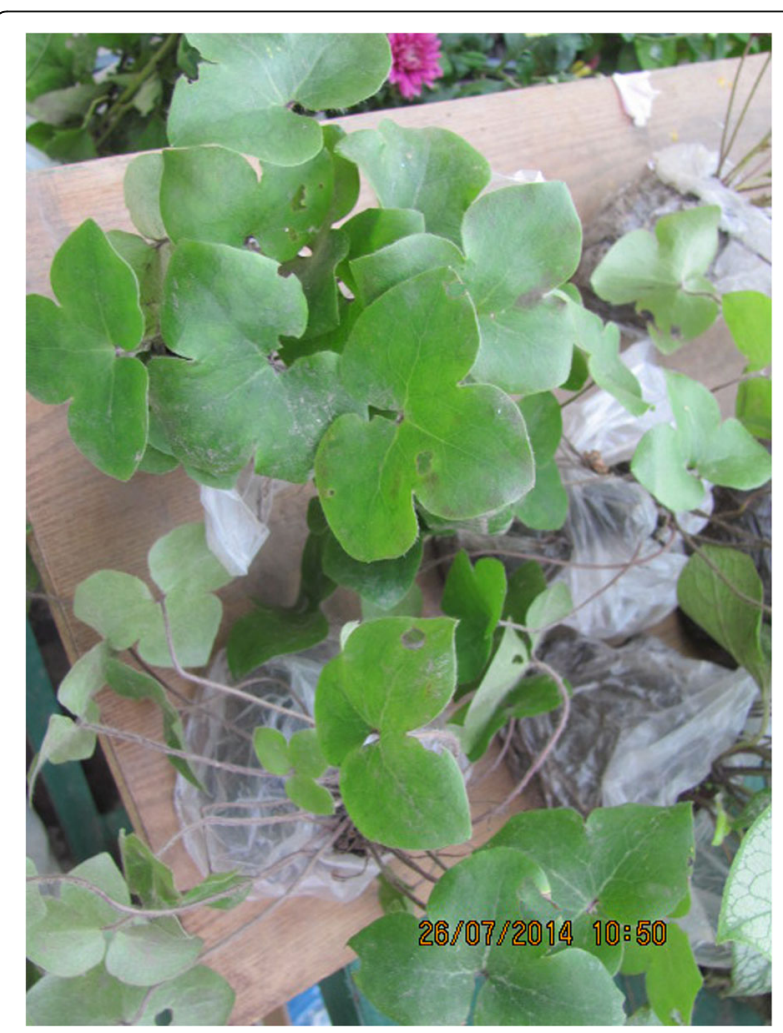

Fig. 9 Wild-origin Hepatica nobilis sold as a garden ornamental in Rzeszów 
Table 1 The results of DNA barcoding

\begin{tabular}{|c|c|c|c|c|c|}
\hline Molecular identification & $\begin{array}{l}\text { Voucher number, starting from } \\
\text { WA00000 }\end{array}$ & $\begin{array}{l}\text { Accession } \\
\text { number }\end{array}$ & $\begin{array}{l}\text { Best match sequence } \\
\text { (accession number) }\end{array}$ & E-value & $\begin{array}{l}\text { Similarity } \\
(\%)\end{array}$ \\
\hline Agaricus bisporus (J.E. Lange) Imbach & 52304 & KX756391 & Agaricus bisporus (LK024175) & 0.0 & 99.56 \\
\hline Armillaria mellea (Vahl) P. Kumm & 52261 & KX756392 & Armillaria mellea (AM269762) & 0.0 & 98.55 \\
\hline Armillaria ostoyae (Romagn.) Herink & 52259 & KX756393 & Armillaria ostoyae (JN657462) & 0.0 & 99.83 \\
\hline \multirow[t]{3}{*}{ Boletus edulis $\mathrm{L}$. } & 52266 & KX756394 & Boletus edulis (KC750230) & 0.0 & 100 \\
\hline & 52295 & KX756395 & Boletus edulis (KC750230) & 0.0 & 100 \\
\hline & 52300 & KX756396 & Boletus edulis (DQ131623) & 0.0 & 99.86 \\
\hline Boletus luridiformis Rostk. & 52272 & KX756397 & Boletus erythropus (UDB001523) & 0.0 & 99.85 \\
\hline Boletus luridiformis Rostk. & 52289 & KX756398 & Boletus erythropus (UDB001523) & 0.0 & 99.85 \\
\hline Chalciporus piperatus (Bull.) Bataille & 52248 & KX756399 & $\begin{array}{l}\text { Chalciporus piperatus } \\
\text { (UDB001528) }\end{array}$ & 0.0 & 98.83 \\
\hline Cortinarius caperatus (Pers.) Fr & 52277 & KX756400 & Cortinarius caperatus (KC842443) & 0.0 & 100 \\
\hline \multirow[t]{8}{*}{ Imleria badia (Fr.) Vizzini } & 52246 & KX756401 & Imleria badia (HM190050) & 0.0 & 99.82 \\
\hline & 52247 & KX756402 & Imleria badia (HM190050) & 0.0 & 98.72 \\
\hline & 52249 & KX756403 & Imleria badia (HM190050) & 0.0 & 99.27 \\
\hline & 52250 & KX756404 & Imleria badia (HM190050) & 0.0 & 100 \\
\hline & 52252 & KX756405 & Imleria badia (HM190050) & 0.0 & 99.82 \\
\hline & 52254 & KX756406 & Imleria badia (HM190050) & 0.0 & 99.36 \\
\hline & 52255 & KX756407 & Imleria badia (HM190050) & 0.0 & 100 \\
\hline & 52263 & KX756408 & Imleria badia (HM190050) & 0.0 & 99.65 \\
\hline Lactarius quieticolor Romagn & 52283 & KX756409 & $\begin{array}{l}\text { Lactarius quieticolor } \\
\text { (UDB001593) }\end{array}$ & 0.0 & 100 \\
\hline \multirow[t]{4}{*}{$\begin{array}{l}\text { Lactarius salmonicolor R. Heim \& } \\
\text { Leclair }\end{array}$} & 52281 & KX756410 & $\begin{array}{l}\text { Lactarius salmonicolor } \\
\text { (DQ679801) }\end{array}$ & 0.0 & 100 \\
\hline & 52296 & KX756411 & $\begin{array}{l}\text { Lactarius salmonicolor } \\
\text { (UDB000370) }\end{array}$ & 0.0 & 100 \\
\hline & 52303 & KX756412 & $\begin{array}{l}\text { Lactarius salmonicolor } \\
\text { (UDB000370) }\end{array}$ & 0.0 & 99.27 \\
\hline & 52305 & KX756413 & $\begin{array}{l}\text { Lactarius salmonicolor } \\
\text { (UDB000370) }\end{array}$ & 0.0 & 99.71 \\
\hline Leccinum schistophilum Bon & 52294 & KX756414 & $\begin{array}{l}\text { Leccinum schistophilum } \\
\text { (UDB019543) }\end{array}$ & 0.0 & 99.10 \\
\hline $\begin{array}{l}\text { Leucoagaricus nympharum (Kalchbr.) } \\
\text { Bon }\end{array}$ & 52299 & KX756415 & $\begin{array}{l}\text { Leucoagaricus nympharum } \\
\text { (JQ683121) }\end{array}$ & 0.0 & 100 \\
\hline Pleurotus cornucopiae (Paulet) Rolland & 52287 & KX756416 & $\begin{array}{l}\text { Pleurotus cornucopiae } \\
\text { (KP877606) }\end{array}$ & 0.0 & 99.53 \\
\hline Polyporus umbellatus (Pers.) Fr. & 52306 & KX756417 & $\begin{array}{l}\text { Polyporus umbellatus } \\
\text { (UDB022812) }\end{array}$ & 0.0 & 99.65 \\
\hline \multirow[t]{2}{*}{ Sparassis crispa (Wulf.) Fr. } & 52290 & KX756418 & Sparassis crispa (KC987583) & 0.0 & 98.94 \\
\hline & 52307 & KX756419 & Sparassis crispa (UDB018795) & 0.0 & 99.48 \\
\hline \multirow[t]{5}{*}{ Suillus bovinus (L.) Roussel } & 52265 & KX756420 & Suillus bovinus (KF482482) & 0.0 & 100 \\
\hline & 52271 & KX756421 & Suillus bovinus (GU016620) & 0.0 & 99.68 \\
\hline & 52282 & KX756422 & Suillus bovinus (GU016620) & 0.0 & 100 \\
\hline & 52288 & KX756423 & Suillus bovinus (GU016620) & 0.0 & 99.68 \\
\hline & 52292 & KX756424 & Suillus bovinus (GU016620) & 0.0 & 100 \\
\hline \multirow[t]{2}{*}{ Suillus grevillei (Klotzsch) Singer } & 52245 & KX756425 & Suillus grevillei (UDB015555) & 0.0 & 99.93 \\
\hline & 52262 & KX756426 & Suillus grevillei (UDB015666) & 0.0 & 99.93 \\
\hline
\end{tabular}


Table 1 The results of DNA barcoding (Continued)

\begin{tabular}{|c|c|c|c|c|c|}
\hline \multirow[t]{7}{*}{ Suillus luteus (L.) Roussel } & 52270 & KX756427 & Suillus luteus (JX907818 & 0.0 & 99.84 \\
\hline & 52274 & KX756428 & Suillus luteus (JX907818) & 0.0 & 100 \\
\hline & 52279 & KX756429 & Suillus luteus (UDB000930) & 0.0 & 100 \\
\hline & 52280 & KX756430 & Suillus luteus (LC035286) & 0.0 & 100 \\
\hline & 52286 & KX756431 & Suillus luteus (JX907818) & 0.0 & 99.69 \\
\hline & 52293 & KX756432 & Suillus luteus (UDB000930) & 0.0 & 99.69 \\
\hline & 52298 & KX756433 & Suillus luteus (JX907818) & 0.0 & 100 \\
\hline Suillus variegatus (Sw.) Kuntze & 52291 & KX756434 & Suillus variegatus (AJ971399) & 0.0 & 100 \\
\hline \multirow[t]{2}{*}{ Tricholoma equestre (L.) P. Kumm. } & 52268 & KX756435 & $\begin{array}{l}\text { Tricholoma equestre } \\
\text { (UDB011389) }\end{array}$ & 0.0 & 100 \\
\hline & 52269 & KX756436 & $\begin{array}{l}\text { Tricholoma equestre } \\
\text { (UDB011389) }\end{array}$ & 0.0 & 100 \\
\hline $\begin{array}{l}\text { Tricholoma frondosae Kalamees \& } \\
\text { Shchukin }\end{array}$ & 52278 & KX756437 & $\begin{array}{l}\text { Tricholoma frondosae } \\
\text { (LT000169) }\end{array}$ & 0.0 & 100 \\
\hline $\begin{array}{l}\text { Xerocomellus cisalpinus (Simonini et al.) } \\
\text { Klofac }\end{array}$ & 52253 & KX756438 & $\begin{array}{l}\text { Xerocomellus cisalpinus } \\
\text { (UDB002180) }\end{array}$ & 0.0 & 99.53 \\
\hline
\end{tabular}

performed using the following thermocycling conditions: an initial $15 \mathrm{~min}$ at $95{ }^{\circ} \mathrm{C}$, followed by 35 cycles at $95{ }^{\circ} \mathrm{C}$ for $30 \mathrm{~s}, 55{ }^{\circ} \mathrm{C}$ for $30 \mathrm{~s}, 72{ }^{\circ} \mathrm{C}$ for $1 \mathrm{~min}$, and a final cycle of 10 min at $72{ }^{\circ} \mathrm{C}$. PCR products were estimated by running $5 \mathrm{ml}$ DNA amplicon on $1.5 \%$ agarose gel for $30 \mathrm{~min}$. The PCR products were sequenced with the use of ITS4 primers, at the Laboratory of Molecular Biology of Adam Mickiewicz University (Poznań). The obtained sequences were verified visually on chromatograms using BIOEDIT. Nuclear ITS sequences obtained in this study are deposited in GenBank [52], with the accession numbers listed in Table 1.

\section{Results and discussion}

\section{General information}

We recorded 468 species of plants sold in the studied markets, including 117 species of plants which are native to Poland, or aliens (anthropophytes) established in the Podkarpacie region (Table 2). However the actual origins of native plants sold in the markets vary and included cultivated plants (84), plants which are both cultivated and collected from the wild (13 species), and species collected only from the wild (19 species).

When only the plants which are at least partly collected from the wild are taken into account, most are sold for food, more rarely for medicinal and ornamental purposes (Fig. 10). When cultivated native plants are added, the ornamental purpose becomes dominant (Fig. 11), followed by food use and medicinal use. There are very few medicinal plants sold solely for this purpose (e.g. Tanacetum vulgae, Hypericum perforatum). Most medicinal plants are fruits used both for food and alcoholic drinks, and as medicine.

As many as 32 species of fungi are sold, all of them for culinary purposes (Table 3), 30 of them are collected from the wild.
Rzeszów is the largest market and hosts the highest biodiversity: 91 species of native or established alien species, compared to 67 in Jarosław, 72 in Leżajsk and 55 in Przemyśl. The largest number of wild-origin species are also sold in Rzeszów (27), compared to 22 in Jarosław, 20 in Przemyśl and 13 in Leżajsk. The largest number of fungi are also sold there (26), compared to 21 in Jarosław, 18 in Przemyśl and 15 in Leżajsk (Table 3).

The interviewed sellers mentioned 46 taxa of plants which they thought had been sold more often in the past, but they mentioned over twice as many (103 taxa, including non-native cultivated plants) as novelties, only recently sold. Out of the species which are sold less, the only wild/ native species was nettle Urtica dioica, mentioned by 2 informants. From the species which are seen as new or increasing, the informants mentioned a few native plants. Predominantly mentioned were ramsons Allium ursinum (5 informants), wormwood Artemisia absinthium (4), sorrel Rumex acetosa (3), Delphinium, blueberry Vaccinium spp. (2), sea buckthorn Hippophae rhamnoides and edelweiss Leontopodium alpinum (Table 4).

\section{Protected plants}

Relatively few protected plants (i.e. protected in the emic sense, according to respondents' knowledge) were listed as sold. Only 12 taxa of protected plants were mentioned by more than one informant. Allium ursinum, Convallaria maialis and Lycopodium were the most often cited. Most of the protected plants were cultivated in gardens (Table 1). This is especially the case with highland plants native only to the highest parts of the Carpathians (Leontopodium alpinum, Dryas octopetala etc.). The species which may be taken from the wild are Allium ursinum, club-mosses and orchids. (Un)fortunately club-mosses were not seen on sale 
Table 2 Plants native to Poland and alien plants which have wild populations in the region sold in south-eastern Poland in open-air markets

\begin{tabular}{|c|c|c|c|c|c|c|c|c|c|c|}
\hline \multirow[t]{2}{*}{$\begin{array}{l}\text { Latin name - taxa which are exclusively or mainly } \\
\text { collected from the wild are written in bold }\end{array}$} & \multirow[t]{2}{*}{$\begin{array}{l}\text { Voucher } \\
\text { specimen } \\
\text { no. } \\
\text { starting } \\
\text { from } \\
\text { WA00000 }\end{array}$} & \multicolumn{4}{|c|}{$\begin{array}{l}\text { Frequency: } 1 \text { - } \\
\text { one seller, 2- } \\
\text { two sellers, } 3 \text { - } \\
\text { more than two } \\
\text { sellers }\end{array}$} & \multirow[t]{2}{*}{ Name used in the market } & \multirow[t]{2}{*}{$\begin{array}{l}\text { Native } \\
\text { status }\end{array}$} & \multirow[t]{2}{*}{ Form of sale } & \multirow[t]{2}{*}{$\begin{array}{l}\text { Origin of } \\
\text { sold plants }\end{array}$} & \multirow[t]{2}{*}{ Purpose of sale } \\
\hline & & $\mathrm{R}$ & $\mathrm{J}$ & $\mathrm{L}$ & $P$ & & & & & \\
\hline Achillea millefolium L. & 52313 & 1 & & & & krwawnik & $\mathrm{R}$ & pots & cult. \& wild & med. \\
\hline Aconitum firmum Rchb. ${ }^{a}$ & & 1 & & & & tojad & $P$ & pots & cult. & orn. \\
\hline Acorus gramineus Sol. ex Aiton & 52314 & 1 & & & & tatarak & A & pots & cult. & orn. \\
\hline Ajuga reptans $\mathrm{L}$. & 52315 & 2 & & 2 & 1 & dąbrówka & $\mathrm{R}$ & pots & cult. & orn. \\
\hline Alchemilla vulgaris $\mathrm{L}$. & 52316 & 2 & 1 & 1 & & przywrotnik & $\mathrm{R}$ & pots & cult. & orn. \\
\hline Allium ursinum $L^{b}{ }^{b}$ & 52317 & 3 & 3 & & 3 & czosnek niedźwiedzi & $\mathrm{R}$ & pots, leaves & cult. \& wild & food, orn. \\
\hline Anemone pulsatilla $\mathrm{L}$. & 52318 & 3 & 3 & 3 & 2 & sasanka & $P$ & pots & cult. & orn. \\
\hline Anemone sylvestris $\mathrm{L}^{\mathrm{b}}$ & 52319 & 1 & 1 & 2 & & zawilec leśny, zawilec biały & $\mathrm{R}$ & pots & cult. & orn. \\
\hline Anemone vernalis $L^{b}$ & & 3 & 3 & 2 & 2 & sasanka & $P$ & pots & cult. & orn. \\
\hline Arabis alpina $\mathrm{L}$. & 52309 & 3 & 1 & 2 & 1 & gęsiówka & $P$ & pots & cult. & orn. \\
\hline Arenaria serpyllifolia L. & 52320 & 2 & & 1 & 1 & piaskowiec & $P$ & pots & cult. & orn. \\
\hline Armeria maritima Willd. & 52321 & 3 & 3 & 3 & & zawciąg & $\mathrm{R}$ & pots & cult. & orn. \\
\hline Armoracia rusticana G. Gaertn. & & 3 & 3 & 3 & 3 & chrzan & A & roots & cult. \& wild & food (spice) \\
\hline Artemisia absinthium L. & 52322 & 3 & 1 & 1 & & piołun & $\mathrm{R}$ & bare rooted plants & cult. \& wild & med. \\
\hline Aruncus dioicus (Walter) Fernald & 52323 & & & 1 & & & $\mathrm{R}$ & pots & cult. & orn. \\
\hline Asarum europaeum L. & 52324 & 1 & & 1 & 1 & kopytnik & r & pots & cult. & orn. \\
\hline Asparagus officinalis $\mathrm{L}$. & 52325 & 1 & & & & szparag & r & bare rooted plants & cult. & food \\
\hline Astrantia major $\mathrm{L}$. & 52326 & & & 1 & & jarzmianka & $r$ & pots & cult. & orn. \\
\hline Bellis perennis $\mathrm{L}$. & 52327 & 3 & 2 & 2 & 1 & stokrotka & r & bouquets, pots & cult. & orn. \\
\hline Berberis vulgaris $\mathrm{L}$. & 52328 & & & 1 & & berberys & r & fruits & cult. & food \\
\hline Calluna vulgaris (L.) Hull & 52329 & 3 & 3 & 3 & 2 & wrzos & $r$ & pots & cult. andwild & orn. \\
\hline Caltha palustris $L$. & 52330 & 1 & & & 1 & kaczyniec, kaczeniec & r & pots & wild & orn. \\
\hline Campanula glomerata $\mathrm{L}$. & & 1 & 1 & 2 & & dzwonek & r & pots & cult. & orn. \\
\hline Campanula persicifolia $\mathrm{L}$. & & 2 & & 2 & 1 & dzwonek & $r$ & pots & cult. & orn. \\
\hline Cardamine glandulifera O.Schwarz & & 1 & & & & - & r & bare rooted plants & cult. & orn. \\
\hline Carlina acaulis L.' & 52331 & 1 & & 1 & & dziewięćsił & r & pots & cult. & orn. \\
\hline Carum carvi L. & 52332 & 1 & & & & kminek & $r$ & pots & cult. & spice \\
\hline Centaurea scabiosa L. & & & & & & - & r & pots & cult. & orn. \\
\hline Convallaria majalis L. & 52333 & 3 & 3 & 2 & 3 & konwalia & $r$ & pots, bouquets & cult. \& wild & orn. \\
\hline
\end{tabular}


Table 2 Plants native to Poland and alien plants which have wild populations in the region sold in south-eastern Poland in open-air markets (Continued)

\begin{tabular}{|c|c|c|c|c|c|c|c|c|c|c|}
\hline$\overline{\text { Corylus avellana } \mathrm{L} \text {. }}$ & & 3 & 3 & 3 & 3 & orzech laskowy & $\bar{r}$ & fruits & cult. \& wild & food \\
\hline Crataegus spp. & & 2 & 1 & & 1 & głóg & r & fruits & wild & food, alc., med. \\
\hline $\begin{array}{l}\text { Crocus vernus (L.) Hill (including Crocus scepusiensis } \\
\text { (Rehm. et Wot. b) }\end{array}$ & & 2 & & & & krokus fioletowy & $\mathrm{p}$ & pots & cult. & orn. \\
\hline Cyanus montanus (L.) Hill. & 52334 & & 1 & & & chaber & r & pots & cult. & orn. \\
\hline Cyanus segetum Hill. & 52335 & 2 & 3 & 2 & 1 & bławatek & a & bouquets, wreaths & wild & orn. \\
\hline Cytisus scoparius (L.) Link & 52336 & 2 & 1 & & & żarnowiec (żółty) & $r$ & pots & cult. & orn. \\
\hline Daucus carota L. & & 3 & 3 & 3 & 3 & marchew & r & roots & cult. & food \\
\hline Delphinium elatum L. & 52337 & 3 & 2 & 3 & 2 & ostróżka & r & pots & cult. & orn. \\
\hline Dianthus carthusianorum L. & & 1 & & & 1 & kartuzek & $r$ & pots & cult. & orn. \\
\hline Dianthus plumarius L. & 52338 & 3 & 1 & 2 & 1 & pierzasty ochr. & $\mathrm{p}$ & pots & cult. & orn. \\
\hline Dictamnus albus L. ${ }^{a}$ & 52339 & 1 & 1 & 1 & & gorejący krzew Mojżesza & $p$ & pots & cult. & orn. \\
\hline Digitalis grandiffora Mill.. & 52340 & 1 & 2 & 1 & & naparstnica & r & pots & cult. & orn. \\
\hline Digitalis purpurea L. & & 3 & 1 & 2 & 1 & naparstnica & a & pots & cult. & orn. \\
\hline Dryas octopetala L. & 52341 & & & 1 & & dębik & $\mathrm{p}$ & pots & cult. & orn. \\
\hline Echinops exaltatus Schrad. & & 2 & & & 1 & przegorzan & a & pots & cult. & orn. \\
\hline Echinops sphaerocephalus L. & 52342 & 1 & & & 1 & przegorzan & a & doniczki & cult. & orn. \\
\hline Eryngium planum L. & 52375 & 1 & & & & mikołajek & r & bukiety & cult. & orn. \\
\hline Euphorbia amygdaloides L. & 52343 & 3 & 2 & 2 & & wilczomlecz & $r$ & pots & cult./wild. & orn. \\
\hline Filipendula vulgaris Moench & 52344 & 1 & & 1 & & wiązówka & $r$ & pots & cult. & orn. \\
\hline Fragaria vesca $\mathrm{L}$. & & 3 & 3 & 2 & 2 & poziomka & r & fruits & cult. & food \\
\hline Galanthus nivalis L. & 52345 & 1 & & & & śnieżyczka & r & bouquet & cult. & orn. \\
\hline Gypsophila paniculata L.' & 52346 & & & 1 & 2 & gipsówka bukietowa & $p$ & pots & cult. & orn. \\
\hline Helianthus tuberosus L. & & 1 & & & & topinambur & a & tubers & cult. & food, ornamental \\
\hline Hepatica nobilis Mill. & 52347 & 2 & 1 & & & przylaszczka & r & pots & cult. and wild & orn. \\
\hline Hypericum perforatum $\mathrm{L}$. & 52348 & 1 & 1 & & 1 & dziurawiec & r & dried aerial parts & wild & med. \\
\hline Inula helenium L. & & & 1 & 1 & & oman & a & pots & cult. & orn. \\
\hline Iris pseudacorus L. & 52349 & 3 & 3 & 3 & 2 & irys & r & pots & cult. & orn. \\
\hline Iris sibirica $\mathrm{L}$. & 52350 & 3 & 2 & 1 & 2 & irys fioletowy & r & pots & cult. & orn. \\
\hline Juniperus communis $\mathrm{L}$. & & & & 1 & & jałowiec & r & pots & cult. & orn. \\
\hline Lamiastrum galeobdolon (L.)L. & & 1 & & & & gajowiec & r & pots & cult. & orn. \\
\hline Ledum palustre L. ${ }^{\mathrm{b}}$ & 52351 & 3 & 3 & 1 & & bagno & r & aerial parts & wild & ins. \\
\hline Leontopodium nivale (Ten.) Huet ex Hand.-Mazz. ${ }^{a}$ & 52352 & 2 & 1 & 1 & & szarotka & r & pots & cult. & orn. \\
\hline Leucanthemum vulgare (Vaill.) Lam. & 52353 & 3 & 3 & 3 & 3 & margaretka/margerytka & r & bukiety, & cult. & orn. \\
\hline
\end{tabular}


Table 2 Plants native to Poland and alien plants which have wild populations in the region sold in south-eastern Poland in open-air markets (Continued)

\begin{tabular}{|c|c|c|c|c|c|c|c|c|c|c|}
\hline Leymus arenarius (L.) Hochst. & & & & 1 & & nadmorska & $p$ & pots & cult. & orn. \\
\hline Ligularia sibirica (L.) Cass. ${ }^{a}$ & & & & 1 & & języczka & $p$ & pots & cult. & orn. \\
\hline Lupinus polyphyllus L. & & 3 & 3 & 2 & & łubin & a & pots & cult. & orn. \\
\hline Lysimachia punctata L. & 52354 & & 1 & 1 & & tojeść & a & pots & cult. & orn. \\
\hline Matricaria chamomilla L. & 52355 & 2 & 1 & 1 & & rumianek & a & inflorescences & cult. & med. \\
\hline Matteucia struthiopteris (L.)Tod. & 52356 & & & & 1 & paprotka & r & pots & cult. & orn. \\
\hline Myosotis sylvatica Hoffm. & 52357 & 2 & & 1 & & niezapominajka & r & pots & cult. & orn. \\
\hline Oenothera sp. & & 1 & & & & wiesiołek & a & pots & cult. & orn. \\
\hline Origanum vulgare L. & 52358 & 3 & 1 & 1 & 1 & oregano & r & pots & cult. \& wild & spice \\
\hline Ornithogalum candicans (Baker) J.C.Manning \& Goldblatt & & & & 1 & & galtonia & r & pots & cult. & orn. \\
\hline Ornithogalum umbellatum $L^{a}{ }^{a}$ & 52310 & 3 & 1 & & 2 & śpioch do 2014 & r & pots & cult. & orn. \\
\hline Pinus sylvestris L. & & 1 & 2 & & 1 & sosna & r & young shoots & wild & alc., med. \\
\hline Polemonium coeruleum L. & & & & & 1 & - & $\mathrm{p}$ & pots & cult. & orn. \\
\hline Polygonatum multiflorum (L.) All. & & & & & 1 & kokoryczka & r & pots & wild & orn. \\
\hline Primula elatior (L.) Hill ${ }^{\mathrm{b}}$ & & 2 & 2 & 1 & & pierwiosnek & r & pots & cult. & orn. \\
\hline Primula veris $\mathrm{L}$. & & & 1 & & & pierwiosnek & r & pots & cult. & orn. \\
\hline Primula vulgaris Huds. & 52359 & 3 & 3 & 3 & 2 & pierwiosnek & r & pots & cult. & orn. \\
\hline Prunus avium L. & & 3 & 3 & 3 & 3 & czereśnia & r & fruits & cult. & food \\
\hline Prunus spinosa L. & & 2 & & & & tarnina & r & fruits & wild & alc. \\
\hline Pulmonaria officinalis L. s.I. & 52360 & 2 & 1 & 2 & & miodunka & r & pots & cult. & orn. \\
\hline Pyrus communis L. & & 3 & 3 & 3 & 3 & gruszka & r & fruits & cult. & food \\
\hline Ribes nigrum L. & & 3 & 3 & 2 & 2 & porzeczka & r & fruits & cult. & food, alc. \\
\hline Ribes uva-crispa L. & & 2 & 2 & 1 & 2 & agrest & r & fruits & cult. & food \\
\hline Rosa canina L. & 52361 & 2 & 2 & & 2 & róża & r & fruits & wild & food, alc., med. \\
\hline Rubus idaeus L. & & 3 & 3 & 3 & 3 & malina & r & fruits & cult. & food \\
\hline Rubus subgenus Rubus & & 3 & 2 & & 1 & ostrężyna & r & fruits & wild & food \\
\hline Rumex acetosa L. & 52362 & 3 & 3 & & 3 & szczaw & r & bundles of leaves & cult. \& wild & food \\
\hline Rumex sanguineus L. & & & & 1 & 1 & - & r & pots & cult. & food \\
\hline Salix caprea L. & 52363 & 3 & 3 & 2 & & bazie & r & leafless twigs with catkins & wild & cer. for Easter \\
\hline Salix of purpurea L. & & & 1 & 1 & & wiklina & r & leafless twigs with catkins, craft material & cult. & cer. for Easter \\
\hline Salvia nemorosa L. & 52311 & 2 & 1 & 1 & & szałwia omszona & $p$ & pots & cult. & orn. \\
\hline Sambucus nigra L. & & 1 & & & & czarny bez & r & racemes of fruits & wild & food, alc., med. \\
\hline Sedum acre L. & 52364 & 2 & 1 & 1 & & rozchodnik & r & pots & cult. & orn. \\
\hline
\end{tabular}


Table 2 Plants native to Poland and alien plants which have wild populations in the region sold in south-eastern Poland in open-air markets (Continued)

\begin{tabular}{|c|c|c|c|c|c|c|c|c|c|c|}
\hline Sempervivum globiferum $L$. & & 1 & & & & rojownik & $r$ & pots & cult. & orn. \\
\hline Silene viscaria (L.) Jess. & 52365 & & 1 & 2 & 1 & smółka & r & pots & cult. & orn. \\
\hline Sorbus aucuparia L. & & 2 & & & 1 & jarzębina & r & fruits & wild & food, alc., med. \\
\hline Staphylea pinnata L. & & 1 & & & & kłokoczka & r & pots & cult. & $\begin{array}{l}\text { orn., religious } \\
\text { (making rosaries) }\end{array}$ \\
\hline Tanacetum parthenium (L.) Sch. Bip. & 52366 & 1 & & 1 & & maruna & a & bouquet & cult. & orn. \\
\hline Tanacetum vulgare $\mathrm{L}$. & 52367 & 1 & & & & wrotycz & r & pots, bundles & wild & med. \\
\hline Thalictrum aquilegifolium L. & & & & 1 & & rutewka & r & pots & cult. & orn. \\
\hline Thymus serpyllum L. & 52368 & 2 & 1 & 1 & 1 & & r & pots & cult. & orn. \\
\hline Tilia cordata Mill. & 52369 & 1 & & & & lipa & r & inflorescences & wild & med. \\
\hline Tripleurospermum maritimum (L.) W. D. J. Koch & & & 1 & 1 & & & a & pots & cult. & orn. \\
\hline Trollius europaeus L. ${ }^{a}$ & & 2 & 2 & & & pełnik & r & pots & cult. & orn. \\
\hline Vaccinium myrtillus $\mathrm{L}$. & & 3 & 3 & 3 & 3 & czarna jagoda & r & fruits & wild & food, med. \\
\hline Vaccinium vitis-idaea $\mathrm{L}$. & & 2 & 2 & 1 & 2 & brusznica & r & fruits & wild & food \\
\hline Valeriana officinalis $\mathrm{L}$. & 52370 & & 1 & & & kozłek & r & pots & cult. & med. \\
\hline Veronica spicata $\mathrm{L}$. & 52371 & 3 & & 1 & 1 & przetacznik & r & pots & cult. & orn. \\
\hline Viburnum lantana L. & & & 1 & & & kalina & $p$ & pots & wild & orn. \\
\hline Viburnum opulus L. & 52372 & 1 & 1 & & 1 & kalina & r & fruits & wild & food, med. \\
\hline Vinca minor $\mathrm{L}$. & 52373 & 1 & & 1 & 1 & barwinek & r & pots & cult. & orn. \\
\hline Viola alba Besser ${ }^{b}$ & & 2 & 1 & 1 & & fiołek & $p$ & pots & cult. & orn. \\
\hline Viola odorata L. & 52312 & 2 & 1 & & 1 & fiołek & a & pots & cult. \& wild & orn. \\
\hline Viola riviniana Rchb. & 52374 & & & & 1 & fiołek & $p$ & pots & cult. & orn. \\
\hline Viscum album L. & & & & 1 & & jemioła & $r$ & aerial parts & wild & cer. for Christmas \\
\hline
\end{tabular}

$R$ Rzeszów, $J$ Jarosław, $L$ Leżajsk, $P$ Przemyśl
$r$ native to the region, $p$ native to Poland but not occurring in the region, $a$ anthropohyte

$r$ native to the region, $p$ native to Poland but not occurring in the region, a anthropohyte
cult. cultivated, orn. ornamental, alc. alcoholic drinks, med. medicinal, ins. insect repellent, cer. ceremonial

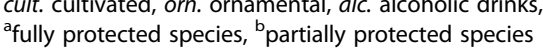




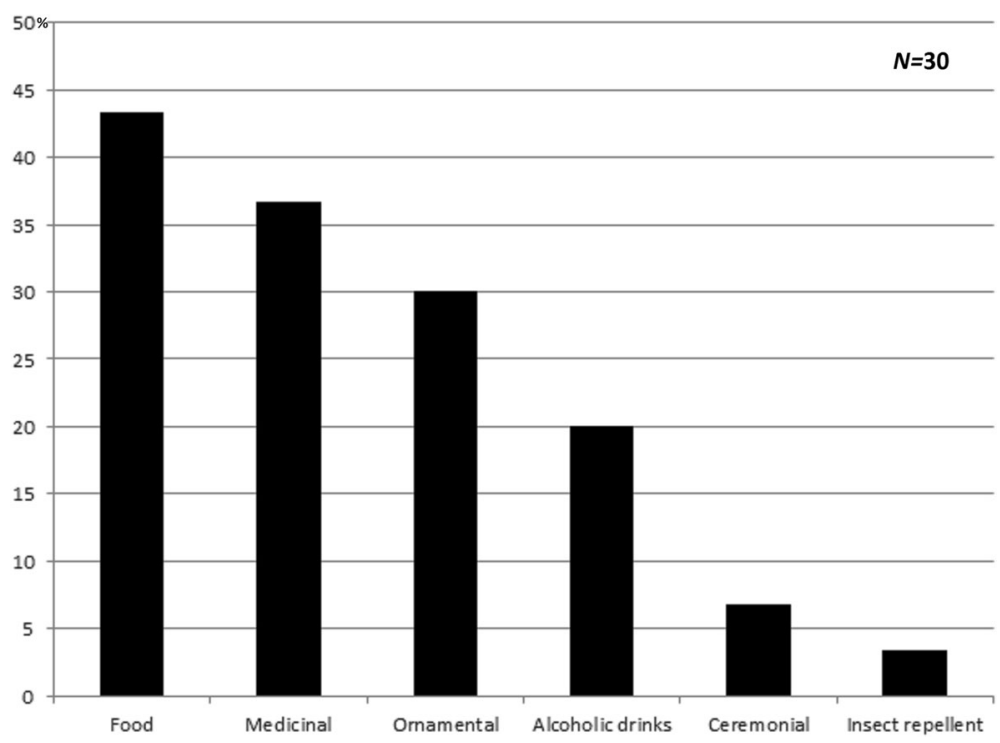

Fig. 10 Use categories for species collected from the wild

in the study period. The sellers of ramsons (Allium ursinum) claim they are cultivated but they have no certificate from local nature conservation authorities, and we suspect that the plants come from wild populations.

Most of the protected plants are sold due to their ornamental value (apart from ramsons, whose use as vegetable has recently become fashionable). Poland is not the only country where plants with beautiful flowers are endangered. For example in Mexico the trade of ornamental orchids creates a biodiversity problem [34].

A very interesting issue is the difference between ordinary people's perceptions of protected plants and which plants are really protected. Our observations allow us to hypothesize that people are over-cautious about plant protection. There are some species which they think are protected but have never been, e.g. Anemone nemorosa. This wild species is common in the woods in Poland and many people think that it is protected by law because they learned at school about the protection of Anemone sylvestris and A. narcissiflora. Recently, in 2014, protection status was removed from many commoner species, which had previously been protected to avoid their being overharvested as medicinal plants (e.g. Frangula alnus, Viburnum opulus,

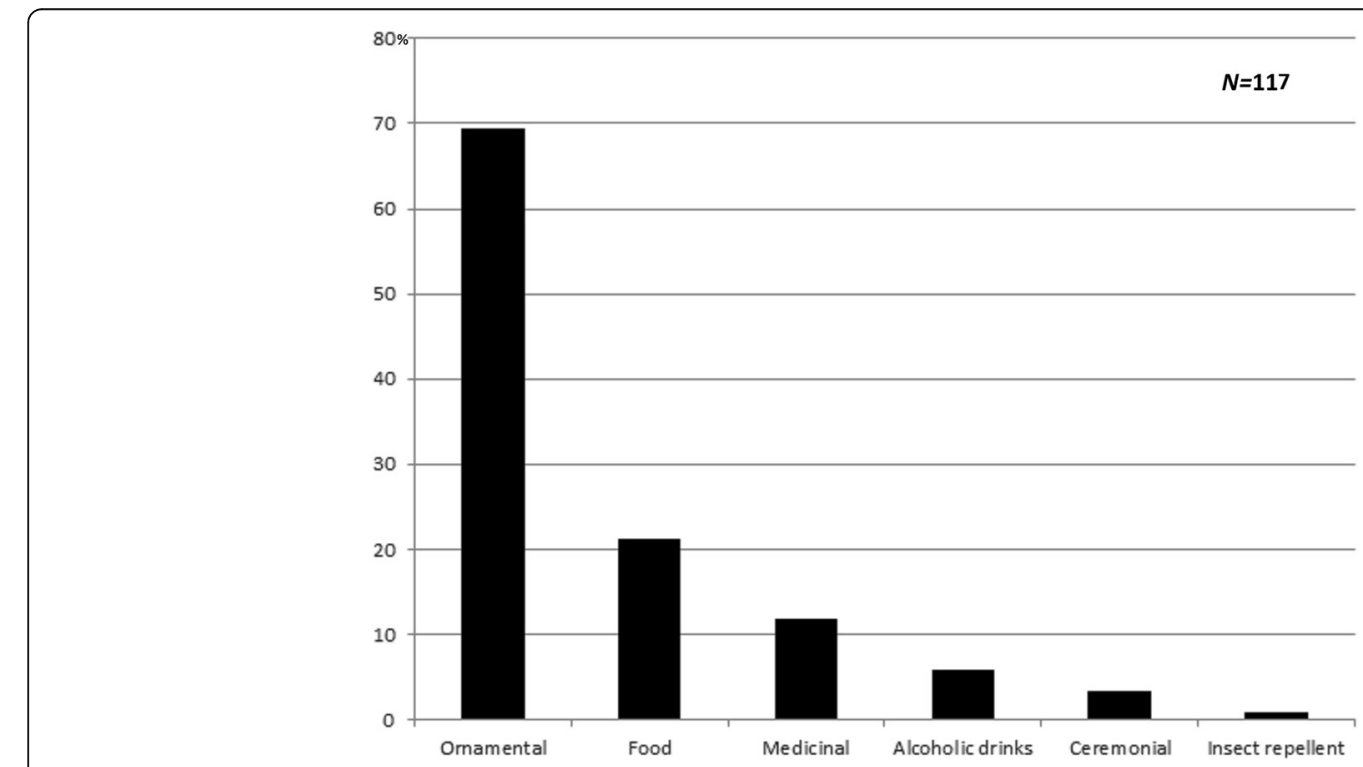

Fig. 11 Use categories for all species which could potentially occur in the wild (native and naturalized) 
Table 3 Edible mushrooms sold in the markets of south-eastern Poland

\begin{tabular}{|c|c|c|c|c|c|c|}
\hline \multirow[t]{2}{*}{ Latin name } & \multirow[t]{2}{*}{ Name used in the market } & \multirow[t]{2}{*}{$\begin{array}{l}\text { Voucher specimen number starting from } \\
\text { WA00000 }\end{array}$} & \multicolumn{4}{|c|}{$\begin{array}{l}\text { Frequency: } \\
1 \text { - one seller, } 2 \text { - two sellers, } 3 \text { - more than two sellers }\end{array}$} \\
\hline & & & Rzeszów & Jarosław & Leżajsk & Przemyś \\
\hline Agaricus bisporus (J.E. Lange) Imbach ${ }^{\text {CULT }}$ & pieczarka & 52304 & 3 & 2 & & \\
\hline Agaricus campestris L. & pieczarka & - & 1 & & & \\
\hline Armillaria gallica Marxm. \& Romagn ${ }^{\#}$ & opieńka & 52284,52308 & 1 & & & \\
\hline Armillaria mellea (Vahl) P. Kumm[ & opieńka & 52261 & 2 & 1 & & 1 \\
\hline Armillaria ostoyae (Romagn.) Herink" & opieńka & 52259 & 3 & 2 & & 2 \\
\hline Boletus edulis $\mathrm{L}$. & prawdziwek & $52266,52295,52300$ & 3 & 3 & 3 & 3 \\
\hline Boletus impolitus Fr." & & 52256 & & 1 & & \\
\hline Boletus luridiformis Rostk." & podciecz, borowik ceglastopory & $52272,52276,52289$ & 2 & 1 & 1 & 1 \\
\hline Boletus subtomentosus L. & zajączek & 52258 & 2 & 3 & & 1 \\
\hline Cantharellus cibarius Fr. & kurka & 52302 & 3 & 3 & 3 & 3 \\
\hline Chalciporus piperatus (Bull.) Bataille ${ }^{1 \#}$ & maślak & 52248 & 1 & & & \\
\hline Cortinarius caperatus (Pers.) Fr & kołpak, chochołka & 52277 & & & 1 & \\
\hline Imleria badia (Fr.) Vizzini & poddąbek, podgrzybek & $52246,52247,52249,52250,52254,52255,52263$ & 3 & 3 & 3 & 3 \\
\hline Lactarius deliciosus (L.) Gray & rydz & 52275 & 3 & 3 & 2 & 1 \\
\hline Lactarius quieticolor Romagn. ${ }^{2 \#}$ & rydz & 52283 & & & 1 & \\
\hline Lactarius salmonicolor R. Heim \& Leclair & rydz & $52281,52296,52303,52305$ & 3 & 2 & 1 & 1 \\
\hline Leccinum aurantiacum (Bull.) Gray & czerwony kozak & $52271,52285,52288,52297$ & 3 & 3 & 1 & 2 \\
\hline Leccinum scabrum (Bull.) Gray & kozak & $52264,52273,52301$ & 3 & 2 & 1 & 2 \\
\hline Leccinum schistophilum Bon ${ }^{3 \#}$ & kozak & 52294 & 1 & & & \\
\hline Leucoagaricus nympharum (Kalchbr.) Bon ${ }^{4 \#}$ & kania & 52299 & 1 & & & \\
\hline Macrolepiota procera (Scop) Singer & kania & 52251 & 3 & 3 & 1 & 2 \\
\hline Pleurotus cornucopiae (Paulet) Rolland $d^{\# C U L T}$ & boczniak & 52287 & 1 & & & \\
\hline Polyporus umbellatus (Pers.) Fr. ${ }^{\text {a\# }}$ & żagiew & 52306 & 1 & & & \\
\hline Sparassis crispa (Wulf.) Fr." & szmaciak & 52290,52307 & & 2 & & 1 \\
\hline Suillus bovinus (L.) Roussel & maślak sitarz & 5265,5282 & 3 & 2 & 1 & 1 \\
\hline Suillus granulatus (L.) Roussel & maślak & $52265,52282,52292$ & 1 & 1 & & 1 \\
\hline Suillus grevillei (Klotzsch) Singer & maślak modrzewiowy & 52245,52262 & 3 & 3 & & 2 \\
\hline Suillus luteus (L.) Roussel & maślak & $52270,52274,52279,52280,52286,52293,52298$ & 3 & 3 & 2 & 3 \\
\hline Suillus variegatus (Sw.) Kuntze & bagniak & 52267,52291 & 2 & 1 & 1 & \\
\hline
\end{tabular}


Table 3 Edible mushrooms sold in the markets of south-eastern Poland (Continued)

\begin{tabular}{lllll}
\hline Tricholoma equestre (L.) P. Kumm. & gąska & 52268,52269 & 3 \\
Tricholoma frondosae Kalamees \& Shchukin\# & gąska & 52278 & 1 \\
Xerocomellus cisalpinus" (Simonini et al.) Klofac & podgrzybek & 52253 & 1 \\
\hline
\end{tabular}

\section{aprotected species}

\#species not present in the official list of mushroom species allowed for sale

'It has peppery taste, confused with Suillus spp

${ }^{2}$ Confused with Lactarius deliciosus or L. salmonicolor, probably an accidental admixture

${ }^{3}$ Confused with Leccinum scabrum, probably an accidental admixture

${ }^{4}$ Confused with Macrolepiota, probably an accidental admixture 
Table 4 "Protected" plants and fungi (in the emic sense) sold in open-air markets according to sellers - regardless of their real protection status. The table includes those mentioned at least twice

\begin{tabular}{|c|c|c|c|c|}
\hline Scientific name & Local name & $\begin{array}{l}\text { No. of } \\
\text { informants }\end{array}$ & $\begin{array}{l}\text { Biogeographical status and } \\
\text { conservation status }\end{array}$ & Origin \\
\hline Allium ursinum $\mathrm{L}$. & $\begin{array}{l}\text { czosnek } \\
\text { niedźwiedzi }\end{array}$ & 11 & $\begin{array}{l}\text { locally abundant in the region } \\
\text { partly protected }\end{array}$ & $\begin{array}{l}\text { mainly collected from the wild, sold in pots and leaves } \\
\text { used as a vegetable }\end{array}$ \\
\hline Convallaria majalis $\mathrm{L}$. & konwalie & 7 & $\begin{array}{l}\text { locally abundant in the region } \\
\text { protected until } 2014\end{array}$ & both from gardens and forests, as cut flowers \\
\hline Lycopodium spp. & widłak & 6 & $\begin{array}{l}\text { locally frequent } \\
\text { partly protected }\end{array}$ & from the wild, not observed on sale \\
\hline Pulsatilla spp. & sasanka & 5 & $\begin{array}{l}\text { practically extinct } \\
\text { protected }\end{array}$ & sold by specialist sellers, from cultivation \\
\hline Galanthus nivalis L. & przebiśnieg & 3 & $\begin{array}{l}\text { abundant but only very locally } \\
\text { partly protected }\end{array}$ & mainly from cultivation \\
\hline Orchidaceae & storczyk & 3 & $\begin{array}{l}\text { locally abundant } \\
\text { protected }\end{array}$ & not observed on sale \\
\hline $\begin{array}{l}\text { Leontoodium } \\
\text { alpinum L. }\end{array}$ & szarotka & 3 & $\begin{array}{l}\text { not native to the region } \\
\text { protected }\end{array}$ & from cultivation \\
\hline Carlina acaulis L. & dziewięćsił & 3 & $\begin{array}{l}\text { rare } \\
\text { partly protected }\end{array}$ & from cultivation \\
\hline Hepatica nobilis L. & przylaszczka & 2 & $\begin{array}{l}\text { locally frequent, protected until } \\
2014\end{array}$ & from cultivation and from the wild \\
\hline $\begin{array}{l}\text { Polygonatum } \\
\text { multifolorum L. }\end{array}$ & kokoryczka & 2 & $\begin{array}{l}\text { frequent, not protected } \\
\text { not protected }\end{array}$ & from cultivation and from the wild \\
\hline Daphne mezereum $\mathrm{L}$. & wawrzynek & 2 & $\begin{array}{l}\text { occasional } \\
\text { partly protected }\end{array}$ & from cultivation and from the wild \\
\hline $\begin{array}{l}\text { Sparassis crispa } \\
\text { (Wulfen) Fr. }\end{array}$ & baraniocha & 2 & frequent, protected until 2014 & from the wild \\
\hline
\end{tabular}

Asarum europaeum, Convallaria majalis), or because we now know that they are more common than previously thought (e.g. Equisetum telmateia).

\section{Edible plants and fungi}

Wild fruits make up an important sector of plants sold in the markets. From the interviews we infer that some species are re-appearing after years of neglect. The main reason for this re-emergence is the growing popularity of herbal medicine and fruit liqueurs. For example such plants as Rosa canina, Crataegus or Prunus spinosa are mainly sold for alcohol production, to a lesser extent also for herbal teas. This trend of the increasing availability of rarer economic plants in Polish market was already noticed by other authors [7, 53].

Mushrooms constitute another important sector of the open-air markets. The number of recorded mushroom species is relatively high. It must be emphasized that the DNA bar-coding we used enabled confirmation of the identification of some surprising taxa sold in the markets (Lactarius quieticolor, Leccinum schistophilum, Leucoagaricus nympharum) never recorded as food in Poland before. Moreover, L. quieticolor and L. schistophilum are not listed in the checklist of Basidiomycetes found in Poland [54] and are new to the mycobiota of Poland. This highlights the importance of DNA barcoding in ethnomycological studies, illustrated well by the study of Dentinger and Suz [55] who found threw new species of porcini (Boletus) in a single packet of mushrooms sold in London and imported from China.

The list of fungi sold in the markets is similar to the taxa reported from a few ethnographic and ethnomycological studies in south-eastern Poland [56]. On the other hand some mushroom taxa widely collected in rural areas are very rarely sold, for example the Russula genus. Russulas can be confused with death cap Amanita phalloides, which is why they are not on the list of taxa permitted for sale in markets $[37,57]$. On the other hand Boletus luridiformis, which is not on the list of taxa allowed for commerce, is more frequently sold (and also traditionally collected here). Altogether 13 species of 32 sold in the markets are not on the list of species legally permitted for sale [57], and even one protected species [58] is sold (Table 3). In some cases very closely related taxa are listed in this legal document (Armillaria, Leccinum), in others the whole genus is not mentioned (even taking into account taxonomic changes and synonyms).

We may conclude that the choice of mushrooms in markets may be a good indicator of culturally salient edible mushrooms, though some lesser collected taxa may not be visible. A similar relationship was found between the traditionally collected wild vegetables in Dalmatia 
and those which are sold in the markets of Dalmatian towns [59], where there is high correlation between the two lists of taxa but some differences occur.

\section{Comparison with other countries}

It is difficult to compare Polish open-air markets with other European countries due to the scarcity of available data, which is restricted to south-eastern Europe. It seems that they have different features from the markets studied in Croatia, Bulgaria and the European part of Turkey. Turkish and Croatian markets sell a large number of wild green vegetables [10-14]. In Poland these are mainly restricted to Rumex species. Bulgarian markets, similarly to the Polish ones, have few wild vegetables, but contain many medicinal plants [13, 14]. Such plants used to be sold in Polish markets in the first half of the 20th century [3-6]. Nowadays purely medicinal plants are rare in Polish markets but the category of culinary herbs is fashionable, however they are mainly nonnatives species. Such species as basil or oregano are relatively new to mainstream Polish cuisine and became fashionable a few years ago. People may be scared to sell purely medicinal plants due to regulations concerning the sales of medicinal material.

\section{Conclusions}

The open-air markets of southeastern Poland sell a considerable number of native plants but only a small proportion of them come from wild populations (mainly edible fruits). Most items are ornamental plants, or edible fruits and mushrooms. Very few medicinal plants and green vegetables are sold, which differentiates the markets from southern European ones. Such a pattern is probably the model for most central European markets, but no similar research has been carried out in neighbouring countries.

Finding two species of fungi which are new to Poland highlights the importance of DNA barcoding in ethnomycological studies.

\section{Acknowledgments \\ Many thanks to prof. Krzysztof Oklejewicz for help in identifying a few plant taxa. \\ Funding \\ The fieldwork was sponsored entirely by the first author. Costs of the analyses and publication were covered by the second and third author's institutions. No external grants and institutions were involved.}

\section{Availability of data and materials}

Voucher specimens of plants and mushrooms are stored in the herbarium of Warsaw University. Photos of plants are available from the first author (R.K-P.) on request. Nuclear ITS sequences obtained in this study are deposited in GenBank [51]. The full lists of species are included in the paper.

\section{Authors' contributions}

RKP performed all the field work and data processing, and identified most of the plants. MP performed DNA barcoding of fungi. LL conceived the methodological framework, identified a few critical taxa, and helped to draft the discussion and write the English version of the paper. All authors read and approved the final manuscript.

\section{Competing interests}

The authors declare that they have no competing interests.

\section{Consent for publication}

Oral prior consent was granted by the interviewees.

\section{Ethics approval and consent to participate}

This kind of research did not need approval from the ethics committees in our faculties.

\section{Author details}

${ }^{1}$ Doctoral Studies Programme, Faculty of Biology and Agriculture, University of Rzeszów, ul. Ćwiklińskiej 2, 35-601 Rzeszów, Poland. ²Department of Plant Taxonomy and Nature Conservation, University of Gdańsk, Wita Stwosza 59, 80-308 Gdańsk, Poland. Institute of Dendrology, Polish Academy of Sciences, ul Parkowa 5, 62-035 Kórnik, Poland. ${ }^{4}$ Department of Botany, Institute of Applied Biotechnology and Basic Sciences, University of Rzeszów, Werynia 502, 36-100 Kolbuszowa, Poland.

Received: 26 August 2016 Accepted: 27 September 2016

Published online: 07 October 2016

References

1. Bye RA. Medicinal plants of the Sierra Madre: comparative study of tarahumara and Mexican market plants. Econ Bot. 1986;40(1):103-24.

2. Nguyen ML, Doherty KT, Wieting J. Market survey research: a model for ethnobotanical education. Ethnobot Res Appl. 2008;17(6):087-92.

3. Muszyński. Wileńskie zioła ludowe. Wiadomości Farmaceutyczne. 1927;21-22:469-76

4. Szulczewski JW. Grzyby sprzedawane na targach Poznania. Rocznik Nauk Rolniczych i Leśnych. 1933;29:1-12.

5. Szulczewski JW. O handlu roślinami leczniczemi na targach w Poznaniu. Poznań: Wydawnictwo Okręgowego Komitetu Ochrony Przyrody w Poznaniu; 1935. p. 80-7

6. Szulczewski JW. Pieśń bez końca: Zbiór tekstów folkorystycznoetnograficznych pod redakcją Wojciecha Łysiaka. Poznań: PSO; 1996.

7. Sucholas J. Zioła i rośliny świąteczne miejskiego targowiska w Poznaniu (Wielkopolska): powrót do badań Szulczewskiego po 80 latach. Herbs and ceremonial plants of the urban marketplace in Poznan (Greater Poland): Szulczewski's study revisited after 80 years. Etnobiologia Polska. 2016;6:7-30.

8. Karousou R, Deirmentzoglou S. The herbal market of Cyprus: traditional links and cultural exchanges. J Ethnopharmacol. 2011;133:191-203.

9. Hanlidou E, Karousou R, Kleftoyanni V, Kokkini S. The herbal market of Thessaloniki ( $N$ Greece) and its relation to the ethnobotanical tradition. J Ethnopharmacol. 2004;91:281-99.

10. Łuczaj Ł, Zovko-Končić M, Miličević T, Dolina K, Pandža M. Wild vegetable mixes sold in the markets of Dalmatia (southern Croatia). J Ethnobiol Ethnomed. 2013;9:2.

11. Ertug F. Wild edible plants of the Bodrum Area (Mugla, Turkey). Turk J Bot. 2004;28:161-74.

12. Dogan Y, Ugulu I, Durkan N. Wild edible plants sold in the local markets of Izmir, Turkey. Pak J Bot. 2013;45(S1):177-84.

13. Nedelcheva A, Dogan Y. An ethnobotanical study on wild medicinal plants sold in the local markets at both sides of the Bulgarian-Turkish border. Planta Medica. 2015;81(16):PW_13

14. Dogan Y, Nedelcheva A. Wild plants from open markets on both sides of the Bulgarian-Turkish border. Ind J Trad Know. 2015;14(3):351-8.

15. Pemberton RW, Lee NS. Wild food plants in South Korea; market presence, new crops, and exports to the United States. Econ Bot. 1996;50(1):57-70.

16. Xu YK, Tao GD, Liu HM, Yan KL, Dao XS. Wild vegetable resources and market survey in Xishuangbanna southwest China. Econ Bot. 2004;58(4):647-67.

17. Liu B, Liu Y, Cao W, Zhang S, Liu Z, Ni Y, Li F. Ethnobotany of Medicinal Aroids in Xishuangbanna, Yunnan Province, China. Aroideana. 2014:69

18. Shirai Y, Rambo AT. Urban demand for wild foods in northeast Thailand: a survey of edible wild species sold in the Khon Kaen municipal market. Ethnobot Res Appl. 2014;12:113-29. 
19. Konsam S, Thongam B, Handique AK. Assessment of wild leafy vegetables traditionally consumed by the ethnic communities of Manipur, northeast India. J Ethnobiol Ethnomed. 2016;12:1.

20. Mati $\mathrm{E}$, de Boer $\mathrm{H}$. Ethnobotany and trade of medicinal plants in the Qaysari Market, Kurdish Autonomous Region, Iraq. J Ethnopharmacol. 2011;133(2):490-510

21. Vlkova M, Verner V, Kandakov A, Polesny Z, Karabaev N, Pawera L, Nadvornikowa I, Banout J. Edible plants sold on marginal rural markets in Fergana Valley, southern Kyrgyzstan. Bulg J Agricult Sci. 2015;21(2):243-50.

22. Hamayun M, Khan MA, Begum S. Marketing of medicinal plants of UtrorGabral Valleys, Swat, Pakistan. Ethnobot Leaflets. 2003;2003(1):13.

23. Amiri MS, Joharchi MR. Ethnobotanical investigation of traditional medicinal plants commercialized in the markets of Mashhad, Iran. Avicenna J Phytomed. 2013;3:254-71

24. Monteiro JM, de Lima Araújo E, Amorim EL, De Albuquerque UP. Local markets and medicinal plant commerce: a review with emphasis on Brazil. Econ Bot. 2010;64(4):352-66.

25. de Albuquerque UP, Monteiro JM, Ramos MA, de Amorim EL. Medicinal and magic plants from a public market in northeastern Brazil. J Ethnopharmacol. 2007;110(1):76-91.

26. Macía MJ, García E, Vidaurre PJ. An ethnobotanical survey of medicinal plants commercialized in the markets of La Paz and El Alto, Bolivia. J Ethnopharmacol. 2005;97(2):337-50.

27. Olowokudejo JD, Kadiri AB, Travih VA. An ethnobotanical survey of herbal markets and medicinal plants in Lagos State of Nigeria. Ethnobotanical Leaflets. 2008;12:851-65.

28. Dibong SD, Ottou PB, Vandi D, Ndjib RC, Tchamaha FM, Mpondo EM. Ethnobotany of anti-hemorrhoidal plants in markets and villages in the central and littoral regions of Cameroon. J Appl Biosci. 2015;96:9072-93.

29. Van Andel T, Myren B, Van Onselen S. Ghana's herbal market. J Ethnopharmacol. 2012;140(2):368-78.

30. Randriamiharisoa MN, Kuhlman AR, Jeannoda V, Rabarison H, Rakotoarivelo N, Randrianarivony T, Raktoarivony F, Randrianasolo A, Bussmann RW. Medicinal plants sold in the markets of Antananarivo, Madagascar. J Ethnobiol Ethnomed. 2015;11(1):1.

31. Ouarghidi A, Powell B, Martin GJ, De Boer H, Abbad A. Species substitution in medicinal roots and possible implications for toxicity of herbal remedies in Morocco. Econ Bot. 2012;66(4):370-82.

32. Kool A, de Boer HJ, Krüger $\AA$, Rydberg A, Abbad A, Björk L, Martin G. Molecular identification of commercialized medicinal plants in Southern Morocco. PLoS One. 2012;7(6):e39459.

33. Martin G. Searching for plants in peasant market-places. In: Plotkin MJ, Famolare L, editors. Sustainable harvest and marketing of rainforest products. Washington, DC: Island Press, Washington; 1992. p. 212-23.

34. Cruz-Garcia G, Lagunez-Rivera L, Chavez-Angeles MG, Solano-Gomez R. The wild orchid trade in a Mexican local market: diversity and economics. Econ Bot. 2015;69(4):291-305.

35. Boa E. Wild edible fungi: a global overview of their use and importance to people. Non-wood forest products 17. Rome: FAO; 2004.

36. Peintner U, Schwarz S, Mešić A, Moreau PA, Moreno G, Saviuc P. Mycophilic or mycophobic? Legislation and guidelines on wild mushroom commerce reveal different consumption behaviour in European countries. PLoS One. 2013;8(5):e63926.

37. Kotowski M. Differences between European regulations on wild mushroom commerce and actual trends in wild mushroom picking. Slovensky Narodopis (Slovak Ethnology). 2016;64(2):169-78.

38. Łuczaj Ł, Stawarczyk K, Kosiek T, Pietras M, Kujawa A. Wild food plants and fungi used by Ukrainians in the western part of the Maramureş region in Romania. Acta Soc Bot Pol. 2015;84(3):339-46.

39. Kang J, Kang Y, Ji X, Guo Q, Jacques G, Pietras M, Łuczaj N, Li D, Łuczaj Ł. Wild food plants and fungi used in the mycophilous Tibetan community of Zhagana (Tewo County, Gansu, China). J Ethnobiol Ethnomed. 2016;12(1):1.

40. Grodzinska K, Godzik B, Szarek-Lukaszewska G. Nature conservation in Poland, emphasising the Carpathian Mountains. In: Szaro RC, Bytnerowicz A, Oszlányi J, editors. Effects of air pollution on forest health and biodiversity in forests of the carpathian mountains, vol. 345. Amsterdam: IOS Press; 2002. p. 59-68.

41. Rozporządzenie Ministra Środowiska z dnia 9 października 2014 r. w sprawie ochrony gatunkowej roślin. http://isap.sejm.gov.pl/DetailsServlet?id= WDU20140001409. Accessed 1 Aug 2016

42. Anonymous. Rozporządzenie Ministra Oświaty z dnia 29 sierpnia $1946 r$. wydane w porozumieniu z Ministrem Rolnictwa i Reform Rolnych i z
Ministrem Leśnictwa w sprawie wprowadzenia gatunkowej ochrony roślin. Dziennik Ustaw. 1946;70:384

43. Eljasz-Radzikowski S. Polscy górale tatrzańscy. Lud. 1897;3:225-72.

44. American Anthropological Association Code of Ethics. http://www.aaanet. org/issues/policy-advocacy/upload/AAA-Ethics-Code-2009.pdf. Accessed 10 Mar 2016

45. International Society of Ethnobiology Code of Ethics(with 2008 additions) http://ethnobiology.net/code-of-ethics/. Accessed 10 Mar 2016

46. Łuczaj Ł. A relic of medieval folklore: Corpus Christi Octave herbal wreaths in Poland and their relationship with the local pharmacopoeia. J Ethnopharmacol. 2012;26;142(1):228-40

47. The Plant List: a working list of all plant species. http://www.theplantlist. org/. Accessed 10 Mar 2016

48. Zając A, Zając M, editors. Atlas rozmieszczenia roślin naczyniowych w Polsce [Distribution Atlas of Vascular Plants in Poland]. Kraków: Jagiellonian University; 2001

49. Index Fungorum. http://www.indexfungorum.org/. Accessed 10 Mar 2016

50. Schocha CL, Seifertb K, Huhndorfc S, et al. Nuclear ribosomal internal transcribed spacer (ITS) region as a universal DNA barcode marker for Fungi. NPAS. 2012;109(16):6241-6.

51. Begerow D, Nilsson $\mathrm{H}$, Unterseher M, Maier W. Current state and perspectives of fungal DNA barcoding and rapid identification procedures. Appl Microbiol Biotechnol. 2010;87:99-108.

52. GenBank. http://www.ncbi.nlm.nih.gov/. Accessed 1 Mar 2016

53. Smalec A. Jarmarki jako forma promocji produktów tradycyjnych. Zeszyty Naukowe Uniwersytetu Szczecinskiego - Problemy Zarządzania Finansów i Marketingu. 2014;35:253-66.

54. Wojewoda W. Checklist of Polish Larger Basidiomycetes. Kraków: W. Szafer Institut of Botany; 2003.

55. Dentinger BT, Suz LM. What's for dinner? Undescribed species in commercial porcini from China. PeerJ. 2014;2:e570.

56. Łuczaj $Ł$, Nieroda Z. Collecting and learning to identify edible fungi in southeastern Poland: age and gender differences. J Nutr Ecol Food Res. 2011;50(4):319-36

57. Rozporządzenie ministra zdrowia z dnia 17 maja 2011 r. w sprawie grzybów dopuszczonych do obrotu lub produkcji przetworów grzybowych, środków spożywczych zawierających grzyby oraz uprawnień klasyfikatora grzybów i grzyboznawcy (Dz.U. 2011 nr 115 poz. 672). http://isap.sejm.gov.pl/ DetailsServlet?id=WDU20111150672\&min=1. Accessed 24 Aug 2016

58. Rozporządzenie Ministra Środowiska z dnia 9 października 2014 r. w sprawie ochrony gatunkowej grzybów. http://isap.sejm.gov.pl/DetailsServlet?id= WDU20140001408. Accessed 1 July 2016.

59. Łuczaj Ł. Fressel N, Perković S. Wild food plants used in the villages of the Lake Vrana Nature Park (northern Dalmatia, Croatia). Acta Soc Bot Pol. 2013;82(4):275-81

\section{Submit your next manuscript to BioMed Central and we will help you at every step:}

- We accept pre-submission inquiries

- Our selector tool helps you to find the most relevant journal

- We provide round the clock customer support

- Convenient online submission

- Thorough peer review

- Inclusion in PubMed and all major indexing services

- Maximum visibility for your research

Submit your manuscript at www.biomedcentral.com/submit
) Biomed Central 